Vol. 2, No. 2, 2017

\title{
MASS MEASURES WITH CODED REMOTE ACCESS FOR CYBER-PHYSICAL SYSTEMS
}

\author{
${ }^{1}$ Bohdan Stadnyk, ${ }^{2}$ Thomas Fröhlich, ${ }^{1}$ Mykola Mykyychuk, ${ }^{3}$ Ihor Klos, \\ ${ }^{1}$ Svyatoslav Yatshyshyn, ${ }^{3}$ Iryna Kalinovska \\ ${ }^{1}$ Lviv Polytechnic National University, 12, S. Bandera str., Lviv, 79013, Ukraine; \\ ${ }^{2}$ Ilmenau University of Technology, 29, Ehrenbergstraße, Ilmenau, 98693, Germany; \\ ${ }^{3}$ Scientific and Production Enterprise "Technobalances", 3, Nadiina str., Lviv, 79013, Ukraine \\ Authors e-mail: slav.yat@gmail.com
}

Submitted on 01.11.2017

(C) Stadnyk B., Fröhlich T., Mykyychuk V., Klos I., Yatshyshyn S., Kalinovska I., 2017

\begin{abstract}
The aspects of design and operation of information measuring subsystem of Cyber-Physical System of coke and chemical plant are considered. This subsystem of mass parameters definition, based on industrial internet, provides coded remote access aiming at the execution of both weighing and verification metrological operations by using an embedded multivalued mass measure. To ensure the quality and reproducibility of Cyber-Physical System a remote access on the basis of TCP/IP protocol to balances was studied and developed. Installation was equipped with the standard built-in mass measure that enabled to perform in-place operations of metrological checking, verification, calibration etc. not interrupting the production cycle.
\end{abstract}

Index Terms: information-measuring subsystem, cyberphysical system, coded remote access, embedded multivalued mass measure.

\section{INTRODUCTION}

Realization of existing Cyber-Physical Systems (further - CPSs) development programs is impossible without taking into account the metrological aspects of their designing, constructing, and operating. CPS technologies companies have to utilize the sophisticated metrology equipment for material and geometric characterization [1]. One of the main types of characterization seems to be the weight determination, and with the lowest values of acceptable errors.

\section{SHORTCOMINGS}

The leading production centers based on modern machinery are not able to be tested by virtue of their own complexity and problems of delivery to certified laboratories. Moreover, unique and newly created machinery often requires self-verification, selfvalidation, and standardization of metrology facilities to ensure the quality operation in a continuous loop. As well, the existing measures lose their accuracy characteristics by several orders while transferring them to the final users, or to the CPSs.

\section{GOAL OF THE WORK}

Aim of the work is studying the possibilities and peculiarities of based on TCP/IP protocol remote monitoring and calibration of balances with embedded mass measure while optimizing their and CPS's metrological characteristics.

\section{THEORETICALAND PRACTICAL FOUNDATIONS OF BALANCE'S REMOTE CALIBRATION}

In current row of articles we have announced earlier [2] about the following consideration of Smart manufacturing, namely in metal production with application of the precise smart balances. For their normal functioning they have to be provided by remote, in-place executable calibration. In this way the creation of remote horizontal-vertical structure of the metrological surveillance of smart instrumentation as the structural units of CPSs, envisages the remote control and verification with using coded access to previously installed software and hardware. Especially it concerns the strain gauge balances applicable in aviation [3], in water control [4] etc.

\section{A. Production with High-Precision Strain Gauge BALANCES}

If complex CPS equipment, in our case the strain gauge balance, is located at great distance from the control device or an information terminal, then it becomes necessary to monitor the state of these units and to manage their characteristics. There are crucially important for balancing their timely calibration, in compliance with their readouts to passport data, because incorrect data usually entail significant financial loss. Therefore, it becomes urgent - the problem of balance's calibration at great distances with the help the industrial Internet [5].

The main requirements for such networks are: the stability of their work in time, rapid transfer of information from system elements, easy network expansion without loss of performance, compatibility with other systems standard protocols, communication over long distance etc.

So, we consider below the aforementioned production cycle equipped with balances (Fig. 1) in the 
presence of their data networks based on TCP/IP protocol. For proper functioning the balance has to be ensured calibration that is performed in its operating place. Due to man-in-loop technology the remotely accreditation of precision balance is accomplished, improving their performance in the certain operating ranges up to working standards values [2]. To implement this technology the multi-level networks and Ethernet are widely applied.

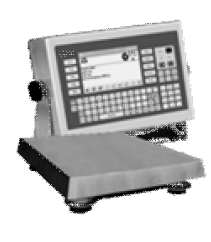

$a$

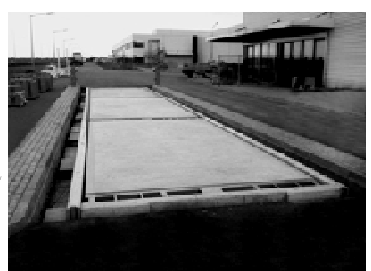

$b$

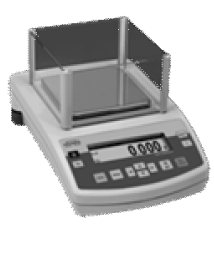

$c$
Fig. 1. Examples of strain gauge weights (a is the platform weight, $b$ is the vehicles weight, $c$ is the lab balance)

Calibration of balance is a set of technical arrangements that establish the mass ratio obtained by balance and standards (or kettlebell) in order to bring metrological characteristics of the first one in compliance with its technical parameters specified in balance's passport details [6]. There exist several types of balance calibration. Initial calibration is performed at balance manufacturing or after repair. Periodic calibration is carried out during the operation when substantial changing of metrological characteristics was fixed after the diagnosis. The same is fulfilled within the terms defined by adjusting calibration intervals which are approved by the results of metrological attestation as it was realized for instance by [7]. Extraordinary calibration is performed while abrupt drifting the balance metrological characteristics was noticed, moreover sooner than calibration interval ended. Manufacturers indicate the recommended periodicity of calibration in their operational documents.

By type of calibration, the balances are divided into the following groups: a) with external calibration (operator performs the calibration with use of kettlebells of appropriate accuracy); with built-in automatic calibration (the need for calibration is determined by the operator, and procedure is carried out by embedded unit); with built-in self-calibration (the need for calibration is determined automatically and performed without operator) [7].

There are several types of networks [8]. In this work the multi-level network for remote calibration of balance with built-in working measure is studied. The information terminal performs role of server. The latter saves information about the state of system elements and manages them. Each of the elements of primary network management may have its subnet in which it is able to act as a server. In every subnet the access to their elements can receive only the network server that enables to split load data processing. Thus, the genera- lized information on system status is transmitted to central control device that sends common control commands.

Until recently the most spread data interface of management information systems was RS-485 imposing several restrictions: number of connected elements not exceed 32, low speed of data transmission, need for creating the separate system network etc.

\section{B. Built-In Weighting SubSyStem With REMOTE ACCESS}

Application of interface Ethernet in management of information subsystems of CPSs units allows to circumvent these restrictions. So, it can transfer the large quantity of information at a greater rate; so it may use the existing networks.

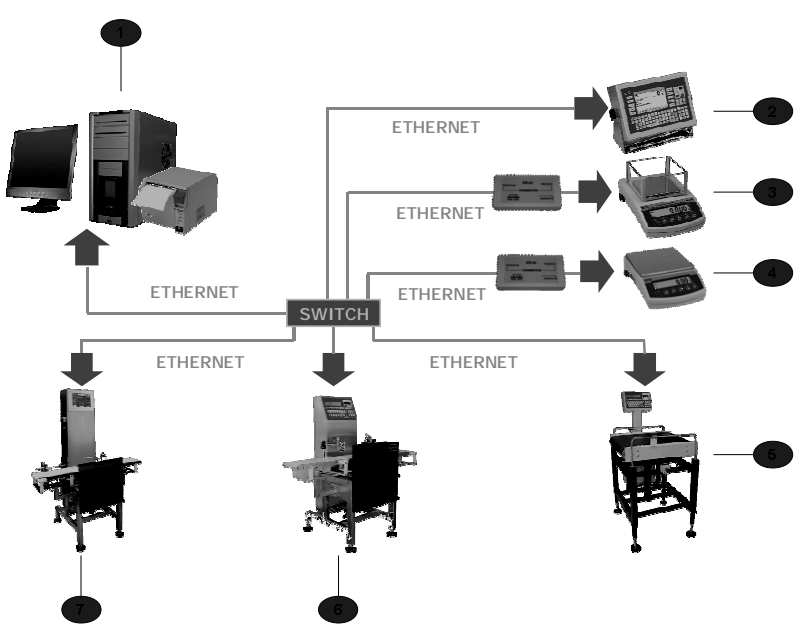

Fig. 2. Block diagram of balances communicating over Ethernet: 1 is the workstation; 2 is the balance-operating microprocessor; 3, 4, 5, 6, 7 are the strain gauge weights of different types

To send information in Ethernet networks there have been worked out a few protocols operating at different levels: application one, transport, gateway level as well as network access layer. The basic network layer protocol of technology TCP/IP is internetwork protocol IP and its subsidiary protocols. This protocol is used to identify the device address of the recipient (in our case particular balance) and the route by which information would be transferred to.

\section{IMPLEMENTATION OF REMOTE CALIBRATION BALANCES WITH BUILT-IN MEASURE}

Having written appropriate software for mentioned system application, we can greatly facilitate the work of operators of weights staff. Then the weights calibration procedures of remote enterprise ("Avdiivka Coke Plant") can be conducted by specialist of Scientific and Production Enterprise "Technobalances", Ukraine, that is located in Lviv, more than $1000 \mathrm{~km}$ from the service recipients. 
The above system is designed to work with car weights. Weighing takes place in two stages that are the gross weighing and the container weighing.

In addition to basic calibration function, this system ensures the transfer of information on vehicles weighing (gross and Tara), multi-level user authentication, prevents unauthorized use of information on carried weighing.

It creates directories with the data: customers, drivers, cargo, warehouses, cars, and vehicle numbers. Also it becomes possible the revision status of weighing, filtering data by specific criteria. In addition, it can search the information on previous weighing, form the common reports for the given period, print the consignment notes, and perform the data archiving. An important component of metrological calibrating procedures can be considered the mass testing, establishing the parameters on a periodic reporting the data of weighing by E-mail.

By continuous controlling the reliability of metrological data and basing on the self-checking results for previous time duration, the forecasting of balance's metrological state is developed. It gives opportunity to correct measurands and to introduce the higher amendments for production parameters.

\section{AVALUATION OF MEASUREMENT ERROR}

Information from output of verified instruments is sent to computing facilities for calculating of errors and creating signal aiming the proper recording of verification results. It forms the basis for the subsequent execution of self-adaptation and self-correction of the performance.

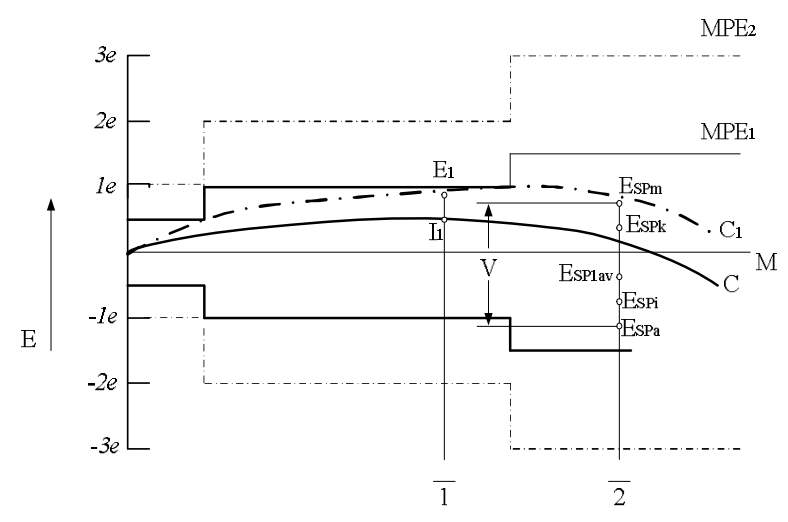

Fig. 3. Terms in usage: $M$ is the value of measured mass, $E$ is the readout error, MRE1 and MRE2 are the maximum permissible errors at initial verification and at operation period respectively, $C$ is the characterization under normal conditions, $\mathrm{Cl}$ is the characteristic caused by impact factor (here is envisaged that impact factor acts as an unchangeable characteristic); ESP is the readout error defined by the longterm instability testing results; I is the basic error; $V$ is the variation of readout error during the long-term instability testing

So, let us consider more consistently the methodological basis of errors' structure of projected measuring instruments intended for weighing in proclaimed goal. Fig. 3 depicts some metrological terms, for defining the basic error, or error in the normal operation mode, etc.

In Fig. 3, cut 1 reflects an error $E_{1}$ of measuring instrument caused by impact factor; I1 is the basic error. Cut 2 displays the errors average value ESPlav for the first measurement during the long-term instability testing as well as some other errors ESPi and ESPk, and errors and boundary values of errors ESPm i ESPn. All these errors should be assessed at different moments of longterm instability testing. At the same time the errors coverage interval $\mathrm{V}$ of readouts during the latter is equal to ESPm - ESPn.

In this method of the remote calibration the considered measuring instrument consists of individual units; each of them is inherent in its own error. While evaluating aforementioned errors need to be applied to the following requirements.

Maximal values of acceptable error $\mathrm{Mi}$, of the certain unit tested separately, are equal to share $p_{i}$ of the high-mentioned error, or permitted variations of readouts of complete measuring instrument device, comprising this unit. Shares for any unit must be chosen according to the same class of exactness and the same number of verification divisions that has a particular measuring instrument that includes this unit.

These shares $p_{i}$ have to meet the following condition:

$$
p_{1}^{2}+\partial_{2}^{2}+\partial_{3}^{2}+\ldots \leq 1
$$

The share $p_{i}$ has to be chosen by unit's manufacturer, and its value must be confirmed by appropriate tests. However, the share should not exceed 0.8 and be not less than 0.3 under more than one unit's action.

Table 1

Shares pi for typical modules

\begin{tabular}{|l|c|c|c|}
\hline & Sensor & $\begin{array}{l}\text { Electronic } \\
\text { indicator }\end{array}$ & $\begin{array}{l}\text { Fastener } \\
\text { s etc }\end{array}$ \\
\hline $\begin{array}{l}\text { The combined } \\
\text { impact* }\end{array}$ & 0.7 & 0.5 & 0.5 \\
\hline $\begin{array}{l}\text { Temperature impact } \\
\text { on readouts without } \\
\text { loading }\end{array}$ & 0.7 & 0.7 & 0.5 \\
\hline $\begin{array}{l}\text { Change of supply } \\
\text { voltage }\end{array}$ & - & 1 & - \\
\hline Impact of drift & 1 & - & - \\
\hline Impact of damp heat & 0.7 & 0.5 & 0.5 \\
\hline
\end{tabular}

* Combined effects of: non-linearity, hysteresis, shortterm effect of temperature. After warming up, the duration of which is set by the manufacturer, constituents of combined impacts error are applicable to modules.

Sign "-“" means "does not apply".

For mechanical structures such as balance's platforms, weighing transmitters, and mechanical or electrical connecting elements that are designed and manufac- 
tured according to the conventional engineering practice, generally apply the share $\mathrm{pi}=0.5$ without any trial. For instance, similar occurs if whole lever is made from the same material and if leverage has two planes of symmetry (longitudinal and transverse), and if characteristics stability of electrical connecting elements corresponds to transmitted signals, such as the sensor output signal, total resistance etc.

For instruments that include typical units given in Table 1, the shares $p_{i}$ can match values specified below.

\section{CONCLUSIONS}

1. To ensure the quality and reproducibility of CPS a remote access on the basis of TCP/IP protocol to such a precise measuring instrument as balances was realized, studied and developed.

2. Moreover installation was equipped with the standard built-in mass measure that enabled to perform onsite operations of metrological checking, verification, calibration etc. without interrupting the production cycle. Consequently, the measure, preset in mentioned complex information-measuring subsystems, is activated distantly by qualified metrologists with help of start-coded signal. Advantage of the proposed method of metrological self-checking is evident and demonstrated on the mass parameters.

3. Possible errors of measurement results were estimated considering that the weighing subsystem

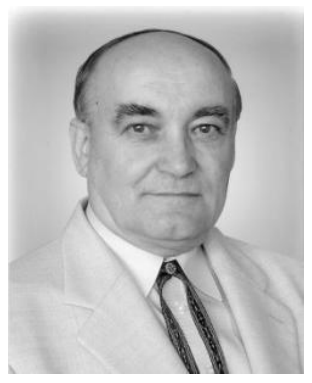

B. Stadnyk, Dr. Sc., Prof., Member of International Thermoelectric Academy, Academician of Ukrainian Academy of Metrology.

Areas of scientific and engineering research: metrology and thermometry, contemporary methods of measurement and standardization, including the intrinsic Standards. Author of 22 books and 493 scientific works.

Areas of scientific and engineering research: metrology and weight measuring equipment, nanotechnology and nanomeasuring machines, standards of physical values. Author of 2 books and 80 scientific works.

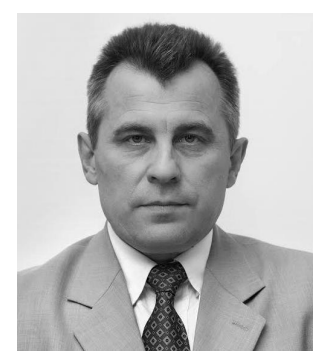

M. Mykyychuk, Dr. Sc., Prof., Academician of Ukrainian Academy of Metrology.

Areas of scientific and engineering research: methods of verification of the measuring tools, solar energetics and its control, means of ensuring the measurement. Author of 2 books and 52 scientific works. consists of individual modules, and each of them shares its own intrinsic error.

\section{ACKNOWLEDGEMENT}

The scientific results, presented in this article, were obtained within the frame of research project number 0115U000446, 01.01.2015-31.12.2017, financially supported by the Ministry of Education and Science of Ukraine.

\section{REFERENCES}

[1] NIST Three-Year Programmatic Plan, FY 2014-2016, 2014.

[2] S. Yatsyshyn, B. Stadnyk, Ya. Luysyk, V. Yatsuk. Metrological Array of Cyber-Physical Systems, Part 1. Challenge of Modernity, Sensors \& Transducers, Vol. 187, Is. 3, 2015, pp. $1-10$.

[3] Strain Gauge Balances for High Load, RUAG Aviation Prospect, Switzerland.

[4] L. Erm, Development of Two-Component Strain-Gauge-Balance Load-Measurement System for the DSTO Water tunnel, DSTOTR-1835, Publ. by Defence Science and Technology Organization, Commonwealth of Australia, 2006.

[5] Industrial Internet Insights Report for 2015, General Electric Company, 2014.

[6] State Standard 3989. Metrology. Calibration of Measuring Instruments, Ukraine, 2000 (in Ukrainian).

[7] Setting and Adjusting Instrument, Calibration Intervals, Agilent Technologies Prospect, 2013.

[8] CSNEN 45501 + AC: Metrological aspects of non-automatic weighing instruments, 1992.

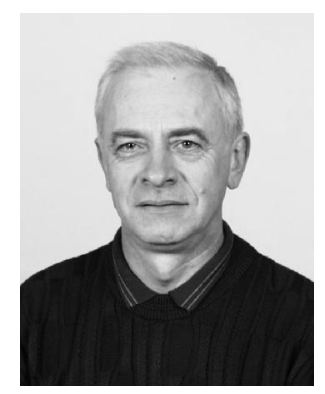

S. Yatsyshyn, Dr. Sc., Prof., Academician of International Thermoelectric Academy, Member of TC-12 of IMEKO, Academician of Ukrainian Academy of Metrology. Areas of scientific and engineering research: temperature measurements, nanometrology and nanothermometry, Standards of physical units. Author of 3 books and 165 scientific works.

I. Klos, Eng., Director of Technobalances Company Ltd, Lviv, Ukraine that is the exclusive representative of the company "Radwag" (Polish manufacturer of weighing equipment) in Ukraine.

T. Fröhlich, Dr. Sc., Extraordinary professor, Associted Member of International Thermoelectric Academy.

I. Kalinovska, Eng., Senior engineer of Technobalances Company Ltd, Lviv. Author of 4 scientific works. 